\title{
Don't Block the Ground: Reducing Discomfort in Virtual Reality with an Asymmetric Field-of-View Restrictor
}

\author{
Fei Wu \\ wuxx1624@umn.edu \\ University of Minnesota \\ Minneapolis, Minnesota, USA \\ Thomas Stoffregen \\ tas@umn.edu \\ University of Minnesota \\ Minneapolis, Minnesota, USA
}

\author{
George Bailey \\ bail0324@umn.edu \\ University of Minnesota \\ Minneapolis, Minnesota, USA \\ Evan Suma Rosenberg \\ suma@umn.edu \\ University of Minnesota \\ Minneapolis, Minnesota, USA
}

\begin{abstract}
Although virtual reality has been gaining in popularity, users continue to report discomfort during and after use of VR applications, and many experience symptoms associated with motion sickness. To mitigate this problem, dynamic field-of-view restriction is a common technique that has been widely implemented in commercial VR games. Although artificially reducing the field-of-view during movement can improve comfort, the standard restrictor is typically implemented using a symmetric circular mask that blocks imagery in the periphery of the visual field. This reduces users' visibility of the virtual environment and can negatively impact their subjective experience. In this paper, we propose and evaluate a novel asymmetric field-of-view restrictor that maintains visibility of the ground plane during movement. We conducted a remote user study that sampled from the population of VR headset owners. The experiment used a within-subjects design that compared the ground-visible restrictor, the traditional symmetric restrictor, and a control condition without FOV restriction. Participation required navigating through a complex maze-like environment using a controller during three separate virtual reality sessions conducted at least 24 hours apart. Results showed that ground-visible FOV restriction offers benefits for user comfort, postural stability, and subjective sense of presence. Additionally, we found no evidence of negative drawbacks to maintaining visibility of the ground plane during FOV restriction, suggesting that the proposed technique is superior for experienced users compared to the widely used symmetric restrictor.
\end{abstract}

\section{CCS CONCEPTS}

- Human-centered computing $\rightarrow$ Virtual reality; • Computing methodologies $\rightarrow$ Virtual reality; Perception.

\section{KEYWORDS}

virtual reality, field-of-view, cybersickness, postural stability

\section{cc) (†)(2)}

This work is licensed under a Creative Commons

Attribution-NonCommercial-ShareAlike International 4.0 License.

SUI '21, November 9-10, 2021, Virtual Event, USA

(C) 2021 Copyright held by the owner/author(s).

ACM ISBN 978-1-4503-9091-0/21/11.

https://doi.org/10.1145/3485279.3485284

\section{ACM Reference Format:}

Fei Wu, George Bailey, Thomas Stoffregen, and Evan Suma Rosenberg. 2021. Don't Block the Ground: Reducing Discomfort in Virtual Reality with an Asymmetric Field-of-View Restrictor. In Symposium on Spatial User Interaction (SUI '21), November 9-10, 2021, Virtual Event, USA. ACM, New York, NY, USA, 10 pages. https://doi.org/10.1145/3485279.3485284

\section{INTRODUCTION}

In recent years, virtual reality (VR) technology has developed rapidly. VR technology has been widely used in entertainment, healthcare, education, and commercial activities. However, many users report discomfort during and after use of VR applications and experience symptoms associated with motion sickness. Users who encounter so-called "cybersickness" may discontinue the experience early or potentially avoid trying a VR system again in the future. Physical discomfort can also reduce task performance and the overall effectiveness of virtual reality applications [28]. Unfortunately, many studies have documented biological sex differences in motion sickness [27], with motion sickness more prevalent among women than among men. This phenomenon therefore introduces important accessibility concerns, especially as immersive technologies become more widely used in work-related contexts.

In a VR application, users' virtual motions may not perfectly match their physical movements in the real world. These discrepancies may be unintentional due to technological factors such as tracking error or system latency, or they may be the result of design decisions made by content creators. For example, virtual locomotion techniques are commonly implemented using handheld controllers to allow movement through virtual environments that are larger than the real world workspace. The resulting mismatch between physical and virtual movements is commonly acknowledged as a major factor that contributes to visually-induced motion sickness in virtual reality.

Dynamic field-of-view (FOV) restriction, also known as "tunneling”, is one of the most popular techniques for mitigating cybersickness and has been widely used in commercial VR games $[4,16]$. This approach partially obscures the user's visual field by displaying a black opaque texture mask in the periphery that dynamically changes size according to movement velocity. Previous researchers have noted a positive correlation between FOV size and VR motion sickness [30]. The FOV restrictor decreases optical flow in the periphery, a perceptual cue widely thought to be associated with 
visually-induced motion sickness. In a formal study, Fernandes and Feiner confirmed that a dynamic FOV restrictor can indeed reduce discomfort during virtual locomotion [16].

Although restricting the field-of-view during movement is a common approach to mitigate cybersickness, it also reduces users' visibility of the virtual environment and can negatively impact their sense of presence [11, 42]. The standard implementation uses a symmetric circular mask to obscure the peripheral FOV, and may or may not include a semi-transparent buffer to smooth the edges of the restrictor. However, the effects of restricting different visual regions have not been sufficiently investigated; and asymmetric restrictors that separate the vertical, horizontal, binocular regions may provide a better cost-to-benefit tradeoff. For example, restricting the lower periphery where the ground plane is displayed may impact the user's postural stability or ability to maneuver around close obstacles, whereas restricting the upper periphery will only mask out portions of the skybox or ceiling.

In this paper, we propose and evaluate a novel variant of FOV restriction, which we refer to as the ground-visible restrictor. This technique uses an asymmetric mask that obscures the upper and side regions of the periphery, but leaves the lower region unoccluded. We then conducted a study to compare the ground-visible restrictor with the standard symmetric FOV restrictor during navigation through a complex maze-like virtual environment. Our results showed that the ground-visible restrictor can improve user comfort during virtual locomotion, and also provides benefits for postural stability and subjective sense of presence. Furthermore, we did not observe any negative effects compared to the traditional symmetric restrictor, and we conclude that the ground-visible technique appears to be a generally superior form of FOV restriction. To the best of our knowledge, this is the first study about the effects of restricting different peripheral regions of the FOV on cybersickness.

\section{RELATED WORK}

\subsection{Motion Sickness in Virtual Reality}

Motion sickness is a natural response of otherwise healthy individuals that can happen in unfamiliar motion environments. Historically, motion sickness has been associated with inertial motions that characterize vehicular travel (e.g., seasickness). Increasingly, motion sickness is a problem with motions that are visual rather than inertial, which are commonplace in video games, virtual environments, VR systems, remotely operated vehicles, and numerous other interactive technologies. In the context of virtual reality, cybersickness has been publicly acknowledged (e.g., by Oculus and other headset manufacturers) as a major limiting factor of contemporary interactive technologies [17].

Cybersickness has similar symptoms to motion sickness, including nausea, oculomotor discomfort, and/or disorientation induced by visual stimuli. Sensory mismatch and postural instability are the commonly postulated causes of cybersickness in virtual reality [39]. The sensory conflict theory claims that motion sickness is caused by a mismatch between current patterns of sensory input about self-motion and expected patterns based on previous experience. In the context of virtual reality, visually induced motion sickness is often associated with virtual imagery that does not correlate with physical motion of the body [19, 28]. The sensation of illusory self-motion in the absence of physical movement, also known as vection, has been discussed in many studies related to visually induced motion sickness [25,37]. According to the postural instability theory, the loss of postural control is not only a consequence but also a causative factor for motion sickness [40]. Differences in postural sway have been observed during exposure to potentially nauseogenic motion in a variety of contexts, such as during stance in a moving room [10], during use of console video games [47], and while wearing a virtual reality headset [34].

\subsection{Mitigating Cybersickness in Virtual Reality}

A variety of methods have been previously proposed to avoid or mitigate symptoms of cybersickness. The first category focuses on addressing technological shortcomings that are associated with visually-induced motion sickness. For example, latency has long been a concern for VR developers, and dynamic variations have been particularly implicated in cybersickness [45], especially at frequencies near $0.2 \mathrm{~Hz}$ [26] [44]. Also, the accommodation/vergence conflict has long been posited to lead to visual discomfort, and some research has found reduced levels of cybersickness when visuals are presented in $2 \mathrm{D}$ rather than in stereo [23].

Although some cybersickness can occur when the user's viewpoint maintains a 1:1 mapping with physical body motion, this phenomenon is most commonly associated with virtual locomotion techniques. A common approach is to avoid continuous visual representations of motion by requiring the user to teleport in the virtual environment [7] [41], although this is often tedious and can lead to diminished spatial awareness [6]. Several variants on this theme have been explored, including "viewpoint snapping" [15], "jumping" [51], "dashing" [32], and "blinking" [20]. Some researchers have noted a reduced incidence of cybersickness when smoothed virtual motions are presented during the virtual traversal of terrain that would normally afford a bumpier trajectory [13]. As another approach to minimizing optical flow cues, it has been proposed to apply a Gaussian blur to the entire presented view during rapid movements [8], to apply a static blur in only the peripheral region [31], to selectively blur pre-defined parts of a scene [35], or to dynamically detect and pre-emptively mask regions of high optical flow in user's visual field [29].

\subsection{Mitigating Cybersickness using FOV Restriction}

As explained in Section 1, dynamic FOV restriction has become one of the most widely used mitigation strategies during virtual locomotion $[4,16]$. This technique is based on the hypothesis that peripheral optical flow is an aggravating factor for cybersickness. FOV restriction has been shown to be effective in mitigating VR sickness for both sexes [2]. However, it should be noted that FOV restriction is not without potential downsides, and current approaches have not proven beneficial under all circumstances [36] [33]. In particular, FOV restriction may not be desirable in the nasal portion of the periphery in the view from each eye, and vertical FOV restriction might be inadvisable to the extent that it occludes the ground plane and horizon line, which people may rely on as a stable reference [21]. Some researchers have instead suggested to employ 
strategies that focus attentional resources on the foveal region without explicitly occluding the periphery [50]. In our current work, the proposed ground-visible restrictor is intended to address these concerns.

Related to the idea of focusing attention away from the periphery, it has long been recognized that stable elements, referred to as "rest frames" may help reduce cybersickness [38] [46]. Varying implementations have been proposed, such as a small reticle superimposed over the center of the visual field [5], a fixed virtual nose [52], a wireframe model of physical world in the periphery [53] and an independent background that fills the entire field of view [14]. While much of this research has been done using rotating drums or projection-based displays, similar effects have also been verified in the context of VR headsets [9]. While the mechanism by which rest frames achieve their sickness-mitigating effects is not yet fully understood [18], both eye movements and postural stability appear to also be affected, as stationary eye fixation also helps to stabilize posture [49]. In addition, the explicit use of passive restraints is a related but somewhat less practical cybersickness mitigation strategy that has been studied in the context of postural stability [24].

\section{FIELD-OF-VIEW RESTRICTOR DESIGN}

The FOV restrictor was implemented in Unity using VR Tunneling Pro, an open-source asset that provides a computionally lightweight dynamic FOV restrictor that can be tuned using a variety of parameters [48]. The industry standard symmetric FOV restrictor was a straightforward use of this asset, as shown in the Figure 1(a). The user's view through each eye is obscured using a circular mask with a black opaque texture in the periphery and a transparent circular hole in the center, and the edges of the opaque region can be optionally smoothed using a semi-transparent effect. The circular cutout is placed at a fixed distance from the center of projection and scales up and down based on the user's velocity, thereby increasing or decreasing the visible FOV.

FOV restrictors are typically displayed only when the participant is moving, and their size is dynamically scaled up to a defined outer radius, which determines how much of the screen is vignetted at maximum motion. An inner radius also defines the intermediate region that transitions linearly between 0 (no opacity) and 1 (full opacity). Although the implementation details vary in different applications, these parameters that are often tunable in VR game menus based on the users' preferences. We attempted to select parameters that were similar to those we observed in commercial games, and conducted internal pilot testing to verify that they provide a comfortable experience. In this experiment, we set the outer radius to 0.60 and the inner radius to 0.1 . Note that these parameters are screen space measurements with respect to the overall FOV; precise angular values are difficult to provide because they will vary based on the optics of individual headsets. The speed of the dynamic scaling was set to 0.25 seconds from non-existence to appearance, or from existence to disappearance.

To create the ground-visible FOV restrictor, we modified the shader to generate a custom texture mask. Instead of rendering the restrictor symmetrically, the horizontal and vertical effects were separated, and new variables were added to control the radius of

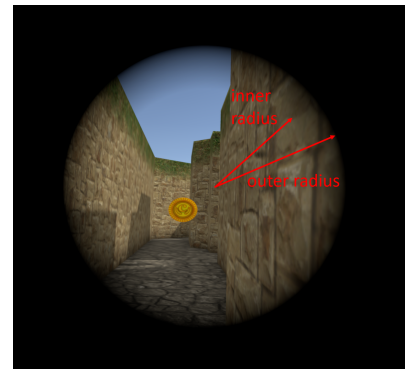

(a)

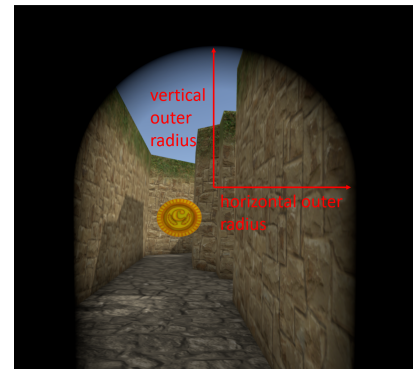

(b)
Figure 1: (a) The standard FOV restriction technique uses a circular black mask to block the periphery of the visual field. (b) The ground-visible restrictor uses an asymmetric mask to preserve view of the lower periphery.

the circular cutout in each direction. The bottom of the vertical effect was then effectively removed by setting the radius to be the maximum vertical FOV of the VR headset. All the other parameters remained consistent with symmetric restrictor. The ground-visible FOV restrictor is shown in the Figure 1(b).

The design of this technique is intended to preserve important perceptual cues provided by the ground plane. It is a metric for egocentric visual space that is used to perceive distance, estimate time-to-collision, and judge the relative size of objects. The optical flow of the observer translating relative to the ground plane can be used to compare spatial intervals [3]. In a study of foveated FOV restriction, which uses an eye tracker to dynamically move the restrictor, results showed that the most frequent area of focus of the participant's eyes was not in the center of the visual field, but rather in the lower region [1]. This suggests that people subconsciously look downwards when walking, and supports the design decision to keep the ground plane visible during movement.

\section{USER STUDY}

\subsection{Experiment Design}

The effectiveness of the ground-visible restrictor was evaluated using a within-subjects design with the following three conditions:

- Symmetric restrictor (S)

- Ground-visible restrictor (GV)

- No FOV restriction (N)

In general, we hypothesized that the ground-visible restrictor would provide the overall best subjective experience for participants across a range of measures, including discomfort, visibility, and sense of presence, and would also exhibit quantitative differences in postural stability metrics derived from motion tracking. Our specific measures and experimental hypotheses are described in more detail in sections 4.5 and 4.6.

The experiment was divided into three virtual reality sessions, one for each condition. Each session lasted 15-20 minutes and was conducted at least 24 hours after the previous session. This was necessary because cybersickness compounds over time and can potentially last for hours; it would therefore not be empirically valid to conduct all three conditions in a single session. The order in which 
each condition was distributed to participants was counterbalanced across the study.

This experiment was originally designed to be conducted in a tightly controlled lab setting. However, due to the COVID-19 pandemic, it was necessary to redesign the study for remote deployment over the internet. This introduced a number of logistical challenges to automate the experiment procedure and required specifically recruiting participants with existing access to consumer virtual reality equipment. The online study protocol was reviewed and approved by our University's Institutional Review Board (IRB).

\subsection{Participants}

Participants in this experiment were recruited through online postings on SteamVR online forums and Reddit interest groups. In the study, participants received study materials through emails and completed each session using their personal equipment. Participants were required to have normal or corrected-to-normal vision and be able to communicate in spoken and written English. Consent materials instructed participants that were pregnant or had a history of epilepsy or severe motion sickness not to participate due to safety concerns. Each participant was compensated with a $\$ 15$ Amazon gift card upon completion of all three virtual reality sessions.

A total of 41 participants completed the study. A total of 38 participants self-identified as male, and only three participants reported as women. Due to prior findings of sex differences in motion sickness, our original study protocol had called for recruiting a balanced sample. However, we discovered that the number of women with access to virtual reality equipment at home remains a very small proportion of the population. This sampling bias is expected to be a major challenge for the virtual reality research community until it becomes possible to safely resume in-person human studies.

Participant ages ranged from 18 to 61 years old $(M=30.5, S D=$ 9.1). Two participants reported that they regularly play video games 0-3 hours per week, eight reported 4-7 hours, 11 reported 8-11 hours, seven reported $12-15$ hours, and 13 reported 16 or more hours per week. Although this would be expected when sampling from a population with access to their own VR equipment, it should be noted that this is a more experienced group than we typically see when recruiting from a university student pool or the general population.

Participants were using their own equipment because the study was conducted remotely due to the COVID-19 pandemic. The following headsets were used in the study: Valve Index (11), HTC Vive (7), HTC Vive Cosmos (1), Oculus Rift S (5), Oculus Rift (4), Oculus Quest with Link Cable (4), and Windows Mixed Reality (9). It should be noted that these devices do have varying technological characteristics such as refresh rate and field-of-view. Because the field-of-view restrictor is defined in screen space (as commonly implemented in commercial VR games), the visible field-of-view displayed using different headsets will vary. In a between-subjects experiment, this could potentially represent a confounding factor. However, the impact of technological differences on the experiment is mitigated by the within-subjects design. Because all participants experienced every experimental condition, we can still empirically compare the relative effects of no restriction, a moderate level

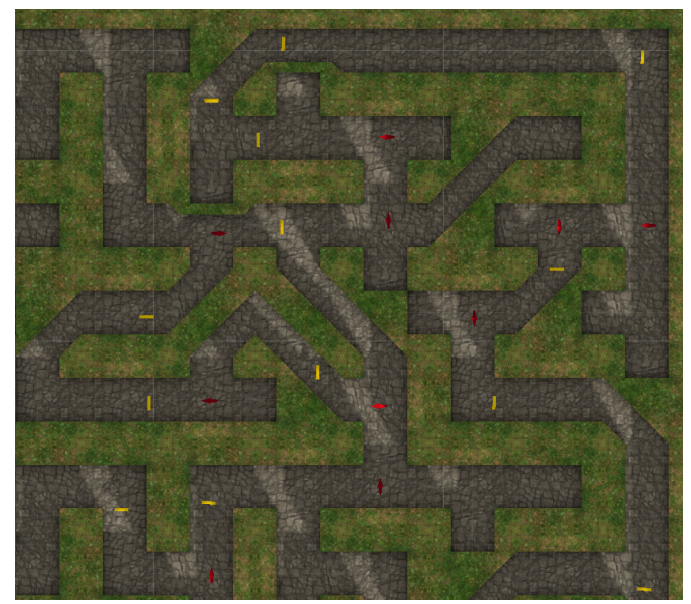

Figure 2: A partial overhead view of the virtual maze. The environment contains complex pathways and frequent turns. The locations of coins and arrows, which are used as waypoints, are indicated in gold and red.

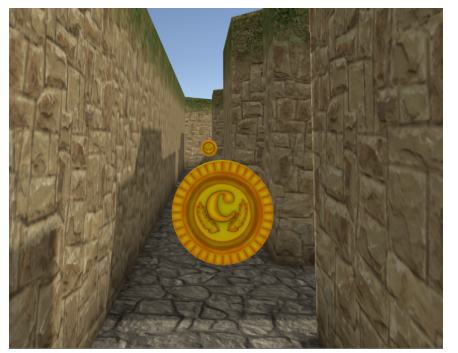

(a)

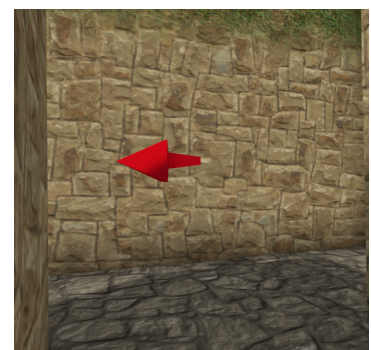

(b)
Figure 3: (a) Participants traversed through the maze and collected gold coins. (b) Red arrows were displayed at crossroads to indicate the correct direction.

of symmetric restriction, and ground-visible restriction, and technological differences between headsets cannot cause a spurious association between the independent and dependent variables.

\subsection{Virtual Environment}

The virtual environment (VE) was designed to be a maze that consists of complex pathways and turns, as shown in Figure 2, which is a close quarter environment. The maze was procedurally generated using an open-source Unity asset [43] and was consistent for all participants. The overall size of the maze was 10 square meters, and the width of each passageway was 1.5 meters. A set of gold coins and red arrows were placed in the maze as waypoints to guide participants through the VE. This was done so that all participants followed the same paths through the maze, because individual exploration strategies would result in different locomotion behavior. Participants were instructed to follow the arrows and collect the gold coins, as shown in Figure 3. When a participant approached a 
coin, it would disappear, and the next one would appear in a readily visible location.

Participants were required to stand during the virtual reality experience. The participants navigated through the VE using a handheld controller. Virtual locomotion was achieved using viewdirected steering, and participants turned using their physical body only. It should be noted that some VR controllers have analog sticks for velocity control, but others only have touchpads, and these input controls have different sensitivity profiles. Therefore, the max velocity was implemented at a rate of 2.5 meters per second in a way that would be consistent across devices. When participants pushed the joystick or clicked the trackpad, the velocity would smoothly accelerate until the max velocity was reached, and then would smoothly decelerate when the joystick/trackpad was released After extensive pilot testing, we determined that implementing smooth acceleration and deceleration over 0.25 seconds provided a good balance between comfort and responsiveness. Because the experimenter could not be physically present to direct the participant if they became lost in the maze, we implemented a function to teleport back to the last waypoint. This could be accessed via a spatial menu activated by the grip button on the controller.

Participants were asked to rate their subjective discomfort at six checkpoints throughout the VR session. We adapted the same procedure from the original FOV restrictor study by Fernandes and Feiner [16], using the question "Please rate the discomfort level you are experiencing right now on a scale from 0 (not discomfort at all) to 10 (severe discomfort)." Responses were provided in the virtual environment using the graphical user interface shown in Figure 4. The participant was instructed to use a laser pointer attached to the controller to select their response, which we refer to as the discomfort score. After completing the checkpoint, the next path would be generated and the participant would be relocated to a different starting point in the maze.

The virtual environment was implemented in Unity 2018.3.6f1 and Steam VR, and was designed to be computationally lightweight with low-poly art assets. During pilot testing using a variety of computers and VR setups, we observed framerates consistently above the headset refresh rate. To verify this after the experiment, we computed the frame rate for each participant from the data files and verified that the virtual environment was rendered at the user's headset refresh rate.

\subsection{Procedures}

Participants initially registered for the study through an online form and subsequently received an email containing the materials for the first session. In the first session, participants reviewed the information sheet and watched an instructional video that explained their tasks and demonstrated how to traverse through the VE using the controller. After starting the application, they completed the Kennedy-Lane Simulator Sickness Questionnaire (SSQ) [22] on their computer monitor using a keyboard and mouse. After completing the SSQ, the application instructed them to put on the headset and follow the instructions to start the virtual reality session.

Each session consisted of a pre-training trial and six experimental trials. Participants would first perform a short practice trial to ensure that they understood the control mechanisms of the virtual

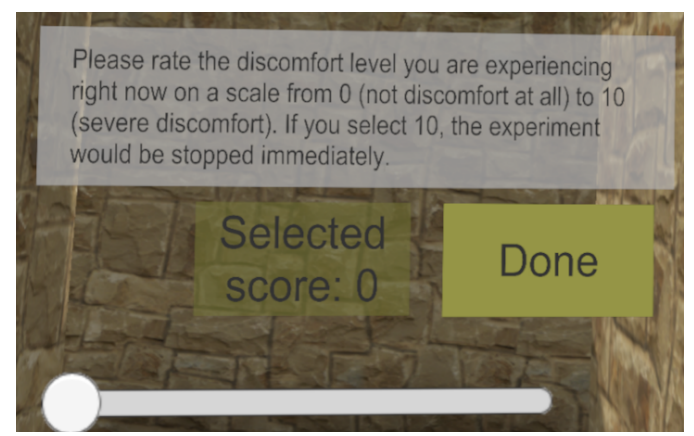

Figure 4: Participants selected a discomfort score using a spatial menu at multiple checkpoints throughout each VR session.

environment. Because the task was limited to just simple movement, and participants were already familiar with using their own VR equipment, the practice trial was brief and could be completed in approximately 30 seconds. After the practice trial, the experiment was started automatically. Each experimental trial required following the path through the maze as described in Section 4.3. This process was designed to last approximately two minutes, although the actual completion time varied between participants. The start and end points for each of the six virtual paths were pre-defined and different for each trial, and the path order was determined pseudo-randomly.

At the end of each trial, participants encountered the checkpoint to capture their discomfort score. Participants were informed in advance that the session would be terminated if they reported a discomfort score of 10 . They could also quit immediately by opening a menu with a controller button and selecting the "quit" option. They were also explicitly instructed in both the written and video materials to discontinue the session if they experienced motion sickness. Otherwise, the session would end automatically when the participant completed the final checkpoint.

Upon completing or terminating the VR session, the participants completed the SSQ post-test and a brief feedback questionnaire. In the third session, the participants also needed to complete a demographic questionnaire at the very end of the experiment. All data collected during the study were automatically compressed into a ZIP file. Participants submitted the data through a link in the email and waited for an email containing the materials for the next session or a gift card to be delivered 24 hours later.

\subsection{Measures}

Discomfort Scores. Similar to the metrics proposed by Fernandes and Feiner [16], we compute two dependent variables from the discomfort scores for each session: the participant's time-weighted Average Discomfort Score (ADS) and the Relative Discomfort Score (RDS). We assume that the discomfort score was 0 at the beginning, and each reported score represents the degree of discomfort before the next checkpoint. The RDS is then computed using the following 
equation:

$$
R D S=\frac{\sum_{0 \leq i \leq t_{\text {stop }}} D S_{i}+\left(t_{\text {max }}-t_{\text {stop }}+1\right) D S_{\text {stop }}}{t_{\max }}
$$

The value $t_{\max }$ represents the longest completion time of all participants. The completion time of each participant was $t_{\text {stop. The }}$ discomfort score at $t_{\text {stop }}$ was recorded as $D S_{\text {stop. }} D S_{i}$ was the discomfort score at each second $i$ prior to $t_{s t o p}$. If a participant terminated before $t_{\text {max }}$, their $D S_{\text {stop }}$ was recorded as 10 and repeated each second from the terminated time until $t_{\max }$. Similarly, if a participant finished early with a discomfort score less than 10, their final score was used as $D S_{\text {stop }}$ and repeated.

Cybersickness. The responses on the Simulator Sickness Questionnaire (SSQ) were used to compute the overall SSQ score before and after each VR session. It should be noted that the SSQ and discomfort scores are not strictly redundant, as they are measuring distinct phenomena. While the SSQ reflects the magnitude of specific symptoms related to cybersickness, the discomfort score encompasses a broader scope that could include sensations not assessed by the SSQ. Additionally, to specifically identify motion sick participants, we also added the following yes/no question on the questionnaire, "Are you motion sick now?"

Subjective Experience. The feedback questionnaire included two sections regarding their experiences in the virtual environment. In the first part, participants were asked to rate their feeling for the three conditions using a 7-point Likert scale from 1="strongly disagree" to $7=$ "strongly agree.":

(1) I felt that my ability to see the virtual environment was restricted.

(2) I had a sense of "being there" in the virtual environment.

For the purposes of readability, the responses for the first question were reversed so that higher ratings are associated with positive outcomes. We referred to these two criteria as visibility and presence, respectively. In the second section of this questionnaire, freeresponse questions were included to gather qualitative comments and suggestions.

Postural Stability. Movement data was recorded automatically by the VR application. During each frame, the system recorded the position and orientation of the participant in the VE, as well as the position and orientation of the participant's head in the physical world. We then computed several variability measures based on only the frames when the participant was moving virtually and the FOV restrictor would be visible. This was done by calculating the standard deviation of the physical movement deltas along a particular degree of freedom ( $\mathrm{x}$-axis, $\mathrm{y}$-axis, roll rotation, and pitch rotation). We adapted these postural stability metrics from motion sickness literature in the field of kinesiology $[12,34]$. For this experiment, we are particularly interested in pitch variability, because the differences between the restrictors could result in different head tilt behavior. If participants were compensating for reduced vertical visibility of the virtual environment when using the $S$ restrictor, increased head tilt variability could contribute to visually-induced motion sickness.
Task Performance. The system also recorded the task completion time and the number of completed trials per participant.

\subsection{Hypotheses}

We formulated six scientific hypotheses regarding the dependent variables collected during this experiment:

- H1: Participants would report lower discomfort scores with the GV restrictor compared to the $\mathrm{N}$ and $\mathrm{S}$ restrictors.

- H2: Participants would report lower SSQ scores with the GV restrictor compared to the $\mathrm{N}$ and $\mathrm{S}$ restrictors.

- H3: Participants would report better visibility with the GV and $\mathrm{N}$ restrictors compared to the $\mathrm{S}$ restrictor.

- H4: Participants would report greater sense of presence with the GV and $\mathrm{N}$ conditions compared to the $\mathrm{S}$ restrictor.

- H5: Postural stability metrics would exhibit greater variability with the $\mathrm{S}$ restrictor compared to the GV and N restrictors.

- H6: Task performance measures would be superior with the $\mathrm{GV}$ and $\mathrm{N}$ restrictors compared to the $\mathrm{S}$ restrictor.

\section{RESULTS}

Shapiro-Wilk tests of normality were conducted for all variables, and the results indicated that none of these data were normally distributed. Because our experiment was within-subjects and nonparametric, we applied the Friedman Rank Test to analyze differences between the three FOV conditions for each dependent variable. Descriptive statistics are therefore reported using median $(M d n)$ and interquartile range $(I Q R)$. All statistical tests used a significance value of $\alpha=0.05$. When a Friedman Rank Test rejected the null hypothesis, we conducted post-hoc analysis using pairwise Conover tests with a Holm-Bonferroni correction for multiple comparisons. One participant had missing data due to accidentally uploading the wrong file, and was therefore excluded from the analysis.

Discomfort Scores. Results for the average and relative discomfort scores are shown in Figure 5. The analysis for ADS revealed significant differences among the three FOV conditions, $\chi^{2}(2)=6.33$, $p=.04$. The GV restrictor $(M d n=0.35, I Q R=0.86)$ was more comfortable than the $\mathrm{N}$ condition $(M d n=0.86, I Q R=1.92), p=.04$. However, ADS ratings for the $\mathrm{S}$ restrictor $(M d n=0.41, I Q R=1.56)$ were not significantly different from the GV restrictor, $p=.36$, or $\mathrm{N}$ condition, $p=.36$. The analysis of RDS was also significant, $\chi^{2}(2)=12.42, p=.002$. Post-hoc comparisons indicated that the $\mathrm{GV}$ restrictor $(M d n=0.44, I Q R=1.03)$ was more comfortable than the $\mathrm{N}$ condition $(M d n=1.01, I Q R=2.32), p=.003$. The $\mathrm{S}$ condition $(M d n=0.69, I Q R=1.62)$ was also more comfortable than $\mathrm{N}$ condition, $p=.03$. RDS ratings for the GV and $\mathrm{S}$ restrictors were not significantly different, $p=.33$. These results partially support hypothesis $\mathrm{H} 1$.

Cybersickness. Analysis of deltas between the pre- and post-SSQ scores did not indicate any significant differences among the three conditions, $\chi^{2}(2)=1.77, p=.41$. Although we found no empirical support for hypothesis H2, SSQ scores were extremely low in all three conditions (see Table 1). Furthermore, participants reported motion sickness on the yes/no question in only 9 out of 120 trials. Taken together, these results suggest a floor effect. 


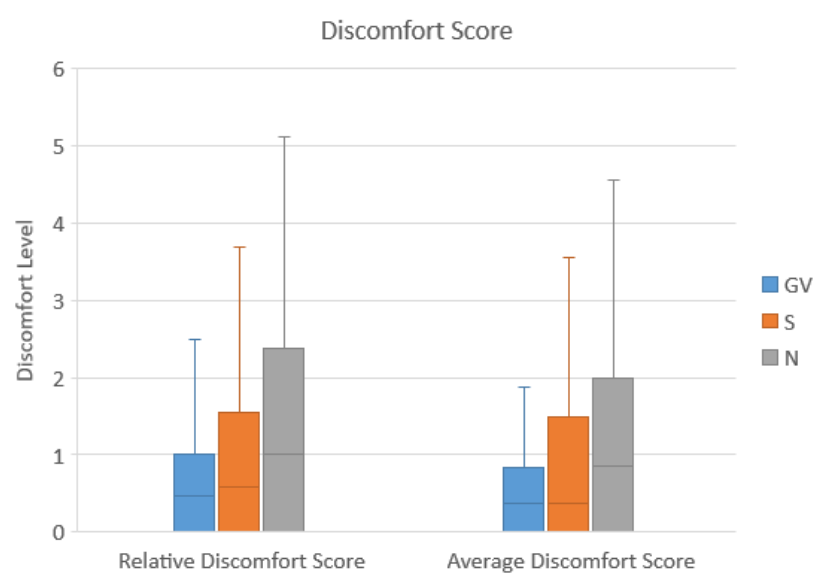

Figure 5: Box plots of the average discomfort score (ADS) and relative discomfort score (RDS) for each condition. Users reported significantly greater discomfort when using the $\mathrm{N}$ restrictor compared to the GV restrictor for both measures.

Table 1: SSQ Scores and Completion Times

\begin{tabular}{lll}
\hline Condition & Mdn SSQ (IQR) & Mdn Completion Time (IQR) \\
\hline N & $7.48(22.44)$ & 784.35 sec. $(130.22)$ \\
S & $7.48(14.96)$ & 750.72 sec. $(89.37)$ \\
GV & $7.48(15.90)$ & 747.60 sec. $(89.56)$ \\
\hline
\end{tabular}

Subjective Experience. Results from the feedback questionnaire are shown in Figure 6. The analysis of visibility ratings revealed a significant difference among the three FOV conditions, $\chi^{2}(2)=$ 58.23, $p<.001$. The $\mathrm{N}$ condition $(M d n=6, I Q R=3)$ was given favorable visibility ratings compared to the $\mathrm{S}$ restrictor $(M d n=1$, $I Q R=1), p<.001$, and the GV restrictor $(M d n=2, I Q R=2)$, $p<.001$. However, there was no significant difference observed between the GV and $\mathrm{S}$ restrictors, $p=.12$. These results partially support hypothesis $\mathrm{H} 3$.

The analysis of presence ratings was also significant, $\chi^{2}(2)=$ 27.37, $p<.001$. Participants reported a greater sense of presence in the $\mathrm{N}$ condition $(M d n=6, I Q R=1.75)$ compared to both the $S$ restrictor $(M d n=5, I Q R=3.75), p<.001$, and GV restrictor $(M d n=5, I Q R=2.75), p=.002$. However, presence ratings for the $\mathrm{GV}$ restrictor were also significantly higher than the $\mathrm{S}$ restrictor, $p=.05$, thereby fully supporting hypothesis $\mathrm{H} 4$.

Postural Stability. Analysis of pitch variability was significant, $\chi^{2}(2)=8.40, p=.02$. Participants exhibited greater pitch variability when using the $S$ restrictor $(M d n=0.16, I Q R=0.08)$ compared to the GV restrictor $(M d n=0.14, I Q R=0.06), p=.02$. The $\mathrm{N}$ condition $(M d n=0.14, I Q R=0.07)$ was not significantly different from either the $\mathrm{S}$ restrictor, $p=.08$, or the GV restrictor, $p=$ .29. Analysis of $\mathrm{x}$-axis variability was also significant, $\chi^{2}(2)=$ $6.91, p=.03$. Participants exhibited greater side-to-side motion variability when using the $\mathrm{S}$ restrictor $(M d n=0.00131, I Q R=$

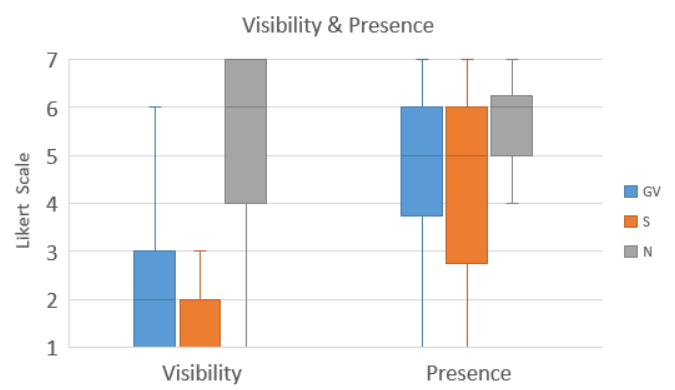

Figure 6: Box plots of visibility and sense of presence ratings for each session. The direction of the scale was adjusted so that higher values are associated with positive outcomes.

$0.00053)$ compared to the GV restrictor $(M d n=0.00130, I Q R=$ $0.00053), p=.03$. The $\mathrm{N}$ condition $(M d n=0.00136, I Q R=0.00057)$ was not significantly different from either the $\mathrm{S}$ restrictor, $p=.39$, or the GV restrictor, $p=.39$. The analysis for z-axis variability was not significant, $\chi^{2}(2)=5.20, p=.07$, nor was roll variability, $\chi^{2}(2)=1.60, p=.45$. These results partially support hypothesis $\mathrm{H} 5$.

Task Performance. Only one participant stopped a VR session early; all others fully completed the six trials in all three conditions. This outlier was therefore excluded from the analysis. Median completion times for each condition are shown in Table 1 . The test did not reveal any significant differences among the three conditions, $\chi^{2}(2)=1.95, p=.37$. We therefore did not find any empirical support for hypothesis H6.

\section{DISCUSSION}

Participants reported the lowest levels of discomfort when using the GV restrictor, as measured through both average and relative discomfort scores. The GV restrictor was the only intervention that significantly improved comfort compared to the condition without restriction. Although the discomfort scores reported when using the GV and S restrictors were not significantly different, we did not find any evidence that would suggest maintaining ground visibility during FOV restriction would be detrimental.

SSQ scores were extremely low overall, which was surprising for a 15-20 minute VR experience that involves controller-based locomotion. These results, along with the very sparse self-reports of motion sickness, suggest a floor effect, and it was unlikely that the restrictors had significant opportunity to further reduce cybersickness. We noted much higher self-reports of cybersickness during pilot testing, and this result was therefore surprising. We therefore cannot make any definitive conclusions regarding the effects of GV restriction on symptoms specific to motion sickness. However, we also note that in the original FOV restrictor study by Fernandes and Feiner [16], SSQ scores were also not significantly different between conditions. Their primary finding of their study involved subjective discomfort ratings, and similar effects were observed in our results.

There are several possible explanations for unusually low cybersickness levels in this experiment. Chief among them is the 
sampling bias inherent in recruiting online participants with preexisting access to VR equipment. Generally speaking, these participants are early adopters, and it stands to reason that people who are more prone to cybersickness would be less likely to invest in a VR setup for personal use. It should also be noted that this population was heavily dominated by men, and we were able to recruit very few women for this study. This is unfortunate due to previous findings that have suggested women are more susceptible to motion sickness compared to men [34]. These issues are very difficult to address during the COVID-19 pandemic, and we plan to conduct follow-up studies that sample from a wider population after it becomes safe to resume in-person experiments. However, we believe that results from studies of active VR users are still valuable, as this population is the primary consumer of virtual reality games where techniques such as FOV restriction are widely used.

To our knowledge, the effects of FOV restriction on postural stability have not been previously evaluated, and this analysis is an additional source of novelty in this experiment. The increased pitch rotation variability when using the S restrictor was likely caused by the need to compensate for reduced vertical visibility. The lower region of the periphery provides perceptual cues that are valuable for close quarters maneuvering, and participants may have needed to tilt their head up and down more often to see imagery that was readily visible when using the GV restrictor. Additionally, the $\mathrm{S}$ restrictor also produced an increase in side-to-side positional variability, which is indicative of postural sway, despite the fact that the left and right regions of the periphery were the same as the GV restrictor. These results suggest that the keeping the ground visible during FOV restriction facilitates stability in control of the body.

It is unsurprising that the condition without restriction was rated highest by participants for both environment visibility and sense of presence. Although the original study by Fernandes and Feiner did not find any significant differences in presence, it should be noted that their experiment used a more conservative FOV restrictor that was designed to be imperceptible to the user [16]. In contrast, we modeled our restrictor design more similarly to implementations commonly present in commercial VR applications such as Skyrim VR or Google Earth VR. In this context, it is notable that the GV restrictor provides benefits for sense of presence compared to $S$ restrictor. Although the visibility score for the GV restrictor was also generally higher, these subjective ratings exhibit a high degree of variability that prevents us from making any definitive scientific conclusions.

Although we had hypothesized that the different conditions would influence navigation task performance, no significant effects were found for completion times. The sampled population, which primarily consisted of experienced users, should again be considered when interpreting these results. We suspect that FOV restriction could have a greater impact on task performance for users that are less familiar with navigation using virtual reality controllers or for people that have greater susceptibility to motion sickness. Therefore, we still consider this to be an interesting question to revisit in future studies.

It is also valuable to consider how the virtual environment design may have influenced the results. In the free response questions, some participants mentioned that the $S$ restrictor sometimes blocked part of the gold coins and red arrows, which led them to stop more often to increase their visibility of the virtual environment. The GV restrictor reduces this problem, which makes it more useful for environments that require close quarters maneuvering. However, for large open environments with objects at a distance, the GV restrictor would have fewer opportunities to provide an advantage. That said, we have not observed any negative effects from using the GV restrictor compared to the $S$ restrictor, and given the potential advantages, we conclude that it appears to be a superior technique for virtual environments that contain a visible ground plane. However, this may not be the case for environments that involve flying through mid-air or open space, and further study would be necessary to evaluate the effects of GV restriction under these conditions.

Finally, as in any study, the scope and generalizability is limited by the specific parameters tested in the experiment. FOV restrictors inherently require a tradeoff between comfort and other aspects of the subjective experience, and the ideal balance is different for every user. In VR applications intended for practical use, we believe that parameters such as restrictor size and speed should be customizable based on the users' individual preferences and tolerances for virtual locomotion.

\section{CONCLUSION AND FUTURE WORK}

In this paper, we proposed and evaluated ground-visible FOV restriction, a novel form of a widely used technique for mitigating cybersickness in virtual reality. We conducted a within-subjects user study across three separate sessions that compared the proposed method with a traditional symmetric restrictor and a control condition without FOV restriction. Our results showed that the ground-visible restrictor offers potential benefits for user comfort, postural stability, and subjective sense of presence. We found no evidence of potential drawbacks to keeping the ground visible during FOV restriction. We therefore conclude that this form appears to be superior to symmetric restrictors when navigating through environments with a visible ground plane. However, this experiment specifically focused on existing VR headset owners, and further study would be needed to generalize these results to naive users or the overall population.

In the future, we plan to further explore the design space for FOV restrictors. This study specifically evaluated the effects during forward and backward locomotion, and these techniques have not been thoroughly investigated during different types of movement, such as turning and side-to-side strafing. It stands to reason that other forms of asymmetric restriction that obscure the left and right periphery in different ways may provide benefits during lateral motion. Additionally, this study was conducted online by recruiting participants with pre-existing access to virtual reality equipment. When it eventually becomes possible to safely resume in-person user studies, we plan to conduct follow-up experiments in a controlled lab setting with a more diverse group of participants and a balanced distribution between the sexes. We also plan to further investigate the relationship between cybersickness mitigation interventions and postural stability, including postural precursors before and during virtual reality exposure. 


\section{ACKNOWLEDGMENTS}

The authors would like to thank Victoria Interrante and Tongyu Nie for their assistance with this research. This material is based upon work supported by the National Science Foundation under Grant No. 1901423.

\section{REFERENCES}

[1] Isayas Berhe Adhanom, Nathan Navarro Griffin, Paul MacNeilage, and Eelke Folmer. 2020. The Effect of a Foveated Field-of-view Restrictor on VR Sickness. In 2020 IEEE Conference on Virtual Reality and 3D User Interfaces (VR). IEEE, 645-652.

[2] Majed Al Zayer, Isayas B Adhanom, Paul MacNeilage, and Eelke Folmer. 2019. The effect of field-of-view restriction on sex bias in vr sickness and spatial navigation performance. In Proceedings of the 2019 CHI Conference on Human Factors in Computing Systems. 1-12.

[3] Jack MH Beusmans. 1998. Optic flow and the metric of the visual ground plane. Vision research 38, 8 (1998), 1153-1170.

[4] Mark Bolas, J Adam Jones, Ian McDowall, and Evan Suma. 2017. Dynamic field of view throttling as a means of improving user experience in head mounted virtual environments. US Patent 9,645,395.

[5] Frederick Bonato, Andrea Bubka, and Wesley W. O. Krueger. 2015. A Wearable Device Providing a Visual Fixation Point for the Alleviation of Motion Sickness Symptoms. Military Medicine 180, 12 (2015), 1268-1272. https://doi.org/10.7205/ MILMED-D-14-00424

[6] Doug A Bowman, David Koller, and Larry F Hodges. 1997. Travel in immersive virtual environments: An evaluation of viewpoint motion control techniques. In Virtual Reality Annual International Symposium. IEEE, 45-52.

[7] Evren Bozgeyikli, Andrew Raij, Srinivas Katkoori, and Rajiv Dubey. 2016. Point \& teleport locomotion technique for virtual reality. In Proceedings of the 2016 Annual Symposium on Computer-Human Interaction in Play. ACM, 205-216.

[8] Pulkit Budhiraja, Mark Roman Miller, Abhishek K Modi, and David Forsyth. 2017. Rotation Blurring: Use of Artificial Blurring to Reduce Cybersickness in Virtual Reality First Person Shooters. arXiv, 1710.02599 (2017). arXiv:1710.02599 http://arxiv.org/abs/1710.02599

[9] Zekun Cao, Jason Jerald, and Regis Kopper. 2018. Visually-induced motion sickness reduction via static and dynamic rest frames. In 2018 IEEE Conference on Virtual Reality and 3D User Interfaces (VR). IEEE, 105-112.

[10] Frank C.Koslucher, Eric J.Haaland, and Thomas A. Stoffregen. 2014. Body load and the postural precursors of motion sickness. Gait \& Posture 39, 1 (jan 2014), 606-610. https://doi.org/10.1016/J.GAITPOST.2013.09.016

[11] James J Cummings and Jeremy N Bailenson. 2016. How immersive is enough? A meta-analysis of the effect of immersive technology on user presence. Media Psychology 19, 2 (2016), 272-309.

[12] Christopher Curry, Nicolette Peterson, Ruixuan Li, and Thomas A Stoffregen. 2020. Postural precursors of motion sickness in head-mounted displays: drivers and passengers, women and men. Ergonomics 63, 12 (2020), 1502-1511.

[13] Jose L. Dorado and Pablo A. Figueroa. 2014. Ramps are better than stairs to reduce cybersickness in applications based on a HMD and a Gamepad. IEEE Symposium on 3D User Interfaces 2014, 3DUI 2014 - Proceedings (2014), 47-50. https://doi.org/10.1109/3DUI.2014.6798841

[14] Henry Been-lirn Duh, Donald E Parker, and Thomas a Furness. 2001. An "independent visual background" reduced balance disturbance envoked by visual scene motion. Proceedings of the SIGCHI conference on Human factors in computing systems - CHI '01 (2001), 85-89. https://doi.org/10.1145/365024.365051

[15] Robert Farmani, Yasin and Teather. 2018. Viewpoint Snapping to Reduce Cybersickness in Virtual Reality. Graphics interface (2018), 159-166. https: //doi.org/10.20380/GI2018.21

[16] Ajoy S Fernandes and Steven K Feiner. 2016. Combating VR sickness through subtle dynamic field-of-view modification. In 2016 IEEE Symposium on 3D User Interfaces (3DUI). IEEE, 201-210.

[17] Tom Forsyth. 2013. VR Sickness, The Rift, and How Game Developers Can Help | Oculus. https://developer.oculus.com/blog/vr-sickness-the-rift-and-how-gamedevelopers-can-help/

[18] Cuiting Guo, Jennifer Ji, and Richard So. 2011. Could OKAN be an objective indicator of the susceptibility to visually induced motion sickness? Proceedings IEEE Virtual Reality (2011), 87-90. https://doi.org/10.1109/VR.2011.5759442

[19] Lawrence J Hettinger and Gary E Riccio. 1992. Visually induced motion sickness in virtual environments. Presence: Teleoperators \& Virtual Environments 1, 3 (1992) 306-310.

[20] M P Jacob Habgood, David Moore, David Wilson, and Sergio Alapont. 2018. Rapid, continuous movement between nodes as an accessible virtual reality locomotion technique. 2018 IEEE Virtual Reality (VR) (2018), 1-9. http://shura.shu.ac.uk/ 18594/

[21] Nupur Kala, Kyungmin Lim, Kwanghyun Won, Jaesung Lee, Tammy Lee, Sehoon Kim, and Wonhee Choe. 2017. P-218: An Approach to Reduce VR Sickness by
Content Based Field of View Processing. SID Symposium Digest of Technical Papers 48, 1 (2017), 1645-1648. https://doi.org/10.1002/sdtp.11956

[22] Robert S Kennedy, Norman E Lane, Kevin S Berbaum, and Michael G Lilienthal. 1993. Simulator sickness questionnaire: An enhanced method for quantifying simulator sickness. The international journal of aviation psychology 3, 3 (1993), 203-220.

[23] Behrang Keshavarz and Heiko Hecht. 2012. Stereoscopic viewing enhances visually induced motion sickness but sound does not. Presence: Teleoperators and Virtual Environments 21, 2 (2012), 213-228. https://doi.org/10.1162/PRES_a_ 00102

[24] Behrang Keshavarz, Alison C. Novak, Lawrence J. Hettinger, Thomas A. Stoffregen, and Jennifer L. Campos. 2017. Passive restraint reduces visually induced motion sickness in older adults. Fournal of Experimental Psychology: Applied 23, 1 (2017), 85-99. https://doi.org/10.1037/xap0000107

[25] Behrang Keshavarz, Bernhard E Riecke, Lawrence J Hettinger, and Jennifer L Campos. 2015. Vection and visually induced motion sickness: how are they related? Frontiers in psychology 6 (2015), 472.

[26] Amelia Kinsella, Ryan Mattfeld, Eric Muth, and Adam Hoover. 2016. Frequency, Not Amplitude, of Latency Affects Subjective Sickness in a Head-Mounted Display. Aerospace Medicine and Human Performance 87, 7 (2016), 604-609. https://doi.org/10.3357/AMHP.4351.2016

[27] Frank Koslucher, Eric Haaland, and Thomas A Stoffregen. 2016. Sex differences in visual performance and postural sway precede sex differences in visually induced motion sickness. Experimental brain research 234, 1 (2016), 313-322.

[28] Joseph J LaViola Jr. 2000. A discussion of cybersickness in virtual environments. ACM Sigchi Bulletin 32, 1 (2000), 47-56.

[29] Jiun-Yu Lee, Ping-Hsuan Han, Ling Tsai, Rih-Ding Peng, Yang-Sheng Chen, Kuan-Wen Chen, and Yi-Ping Hung. 2017. Estimating the simulator sickness in immersive virtual reality with optical flow analysis. SIGGRAPH Asia 2017 Posters on - SA '17 (2017), 1-2. https://doi.org/10.1145/3145690.3145697

[30] JJ-W Lin, Henry Been-Lirn Duh, Donald E Parker, Habib Abi-Rached, and Thomas A Furness. 2002. Effects of field of view on presence, enjoyment, memory, and simulator sickness in a virtual environment. In Proceedings IEEE Virtual Reality 2002. IEEE, 164-171.

[31] Yun-Xuan Lin, Rohith Venkatakrishnan, Roshan Venkatakrishnan, Elham Ebrahimi, Wen-Chieh Lin, and Sabarish V Babu. 2020. How the Presence and Size of Static Peripheral Blur Affects Cybersickness in Virtual Reality. ACM Transactions on Applied Perception (TAP) 17, 4 (2020), 1-18.

[32] Sysdia Solutions Ltd. $2019 . \quad$ VRTK_DashTeleport. https://vrtoolkit.readme.io/docs/vrtk dashteleport. Accessed on 2019-09-27.

[33] J.D. D Moss and E.R. R Muth. 2011. Characteristics of head mounted displays and their effects on simulator sickness. Human Factors 53, 3 (2011), 308-319. https://doi.org/10.1177/0018720811405196.Copyright

[34] Justin Munafo, Meg Diedrick, and Thomas A Stoffregen. 2017. The virtual reality head-mounted display Oculus Rift induces motion sickness and is sexist in its effects. Experimental brain research 235, 3 (2017), 889-901.

[35] Guangyu Nie, Yue Liu, and Yongtian Wang. 2017. Prevention of Visually Induced Motion Sickness Based on Dynamic Real-Time Content-Aware Non-salient Area Blurring. Adjunct Proceedings of the 2017 IEEE International Symposium on Mixed and Augmented Reality, ISMAR-Adjunct 2017 (2017), 75-78. https://doi.org/10. 1109/ISMAR-Adjunct.2017.35

[36] Nahal Norouzi and Greg Welch. 2018. Assessing Vignetting as a Means to Reduce VR Sickness During Amplified Head Rotations. ACM Symposium on Applied Perception (2018).

[37] Stephen Palmisano, Robert S Allison, Mark M Schira, and Robert J Barry. 2015. Future challenges for vection research: definitions, functional significance, measures, and neural bases. Frontiers in psychology 6 (2015), 193.

[38] Jerrold Douglas Prothero. 1998. The Role of Rest Frames in Vection, Presence and Motion Sickness. Ph.D. Dissertation. University of Washington, Seattle.

[39] Lisa Rebenitsch and Charles Owen. 2016. Review on cybersickness in applications and visual displays. Virtual Reality 20, 2 (2016), 101-125.

[40] Gary E Riccio and Thomas A Stoffregen. 1991. An ecological theory of motion sickness and postural instability. Ecological psychology 3, 3 (1991), 195-240.

[41] Roy A. Ruddle, Andrew Howes, Stephen J. Payne, and Dylan M. Jones. 2000. Effects of hyperlinks on navigation in virtual environments. International fournal of Human Computer Studies 53, 4 (2000), 551-581. https://doi.org/10.1006/ijhc. 2000.0402

[42] A Fleming Seay, David M Krum, Larry Hodges, and William Ribarsky. 2001. Simulator sickness and presence in a high FOV virtual environment. In Proceedings IEEE Virtual Reality 2001. IEEE, 299-300.

[43] Sergey Smurov. 2018. QMaze. https://assetstore.unity.com/packages/tools/ modeling/qmaze-30600/. Accessed on 12-01-2020.

[44] Matthew E. St. Pierre, Salil Banerjee, Adam W. Hoover, and Eric R. Muth. 2015. The effects of $0.2 \mathrm{~Hz}$ varying latency with $20-100 \mathrm{~ms}$ varying amplitude on simulator sickness in a helmet mounted display. Displays 36 (2015), 1-8. https://doi.org/ 10.1016/j.displa.2014.10.005

[45] Jan-Philipp Stauffert, Florian Niebling, and Marc Erich Latoschik. 2018. Effects of Latency Jitter on Simulator Sickness in a Search Task. IEEE Virtual Reality 
(2018), 121-127.

[46] R. M. Stern, S. Hu, R. B. Anderson, H. W. Leibowitz, and K. L. Koch. 1990. The effects of fixation and restricted visual field on vection-induced motion sickness Aviation, Space and Environmental Medicine 61, 8 (1990), 712-715.

[47] Thomas A. Stoffregen, Elise Faugloire, Ken Yoshida, Moira B. Flanagan, and Omar Merhi. 2008. Motion Sickness and Postural Sway in Console Video Games. Human Factors: The fournal of the Human Factors and Ergonomics Society 50, 2 (apr 2008), 322-331. https://doi.org/10.1518/001872008X250755

[48] Luke Thompson. 2017. VR Tunnelling Pro. http://www.sigtrapgames.com/ VrTunnellingPro/html/. Accessed on 12-01-2020.

[49] Nicholas A. Webb and Michael J. Griffin. 2003. Eye movement, vection, and motion sickness with foveal and peripheral vision. Aviation Space and Environmental Medicine 74, 6 (2003), 622-625.
[50] Yue Wei, Jiayue Zheng, and Richard H. Y. So. 2018. Allocating less attention to central vision during vection is correlated with less motion sickness. Ergonomics 61, 7 (2018), 933-946.

[51] Tim WeiBker, Kunert Bernd, F Ohlich, and Alexander Kulik. 2018. Spatial Updating and Simulator Sickness during Steering and Jumping in Immersive Virtual Environments. Ieee $\operatorname{Vr}$ (2018), 256-307.

[52] Carolin Wienrich, Christine Katharina Weidner, Celina Schatto, David Obremski, and Johann Habakuk Israel. 2018. A virtual nose as a rest-frame-the impact on simulator sickness and game experience. In 2018 10th international conference on virtual worlds and games for serious applications (VS-Games). IEEE, 1-8.

[53] Fei Wu and Evan Suma Rosenberg. 2019. Combining dynamic field of view modification with physical obstacle avoidance. In 2019 IEEE Conference on Virtual Reality and 3D User Interfaces (VR). IEEE, 1882-1883. 